\title{
Percutaneous nephrolithotomy: keeping the bridge for one night
}

\author{
Cengiz Kara
}

Received: 18 November 2011 / Accepted: 29 November 2011/Published online: 19 January 2012

(C) Springer-Verlag 2012

We read the retrospective study comparing the outcomes of nephrostomy-free percutaneous nephrolithotomy (PCNL) with early nephrostomy tube removal (after 1 day) with great interest [1]. Only 55 highly selected cases who underwent non-complicated PCNL through a single tract were identified to receive either no nephrostomy tube or an 22-Fr nephrostomy tube. They stated that in the tubeless group, all patients were administered with a ureteral catheter for drainage of the kidney. Preoperatively, the groups were similar. Postoperatively, a large proportion of patients in each group were stone-free. Post-operative events such as hematuria and renal colic were significantly more in tubeless group. Mean dose of post-operative analgesia, mean hemoglobin deficit and hospital days were comparable for both groups. The study has shown that 1-day nephrostomy technique after PCNL is significantly better post-operative course and it is comparable to nephrostomyfree technique in analgesic requirements and hospital stay. The nephrostomy tube also provides a bridge for second look nephroscopy in patients with residual stones.

With the intent of reducing post-operative discomfort and pain, hospital stay, and cost, tubeless PCNL procedure has gained popularity in recent years [2].

Traditionally, the placement of a nephrostomy tube at the conclusion of PCNL is considered the standard of care. However, the need for nephrostomy tube placement has been questioned by numerous authors. In two recent metaanalysis with 90 studies, it was shown that tubeless PCNL was a safe and effective procedure for treatment of large renal stones in selected patients with shorter hospital stay,

C. Kara $(\square)$

Ataturk Egitim ve Arastirma Hastanesi, 1. Uroloji Klinigi,

Basınsitesi, 35360 Izmir, Turkey

e-mail: drcengizkara@hotmail.com less analgesic requirement, lower urine leakage and without increased complications [3, 4]. On the other hand, appropriate patients should be selected for tubeless PCNL. We believe that, this procedure will only be acceptable when safety has not been sacrificed. For this reason, we use criteria such as minimal bleeding, no residual stone and intact collecting system when choosing the patients in our center. Renal colic was observed in four patients after the operation in tubeless group in this study. It is not surprising to see that observation of ureteral stents may cause mucosal irritation. We believe that the major advantage for patients undergoing tubeless PCNL was the absence of stent-related flank pain and dysuria.

We agree with the authors comment that the possible limitation of the tubeless PCNL is that it precludes second look PCNL for residual stones. However, when a secondary percutaneous procedure for removal of residual stones is planned, the pre-existing tract may not provide good access to reach the stone. In most cases, generating a new access tract may be necessary. Alternatively, residual calculi can be safely managed by SWL or retrograde intrarenal surgery in this group of patients.

\section{References}

1. El-Nahas AR, Shokeir AA (2011) Percutaneous nephrolithotomy: keeping the bridge for one night. Urol Res [Epub ahead of print]

2. Kara C, Resorlu B, Bayindir M, Unsal A (2010) A randomized comparison of totally tubeless and standard percutaneous nephrolithotomy in elderly patients. Urology 76(2):289-293

3. Zilberman DE, Lipkin ME, de la Rosette JJ et al (2010) Tubeless percutaneous nephrolithotomy-the new standard of care. J Urol 184:1261-1266

4. Yuan H, Zheng S, Liu L et al (2011) The efficacy and safety of tubeless percutaneous nephrolithotomy: a systematic review and meta-analysis. Urol Res 39(5):401-410 